\title{
SCHWARZ REFLECTION PRINCIPLES FOR SOLUTIONS OF PARABOLIC EQUATIONS ${ }^{1}$
}

\author{
DAVID COLTON
}

\begin{abstract}
A reflection principle is obtained for solutions of the heat equation defined in a cylindrical domain of the form $\Omega \times(0, T)$ where $\Omega$ is a ball in $R^{n}$ and the solution vanishes on $\partial \Omega \times(0, T)$. It is shown that the domain of dependence of the solution at a point outside the cylinder $\Omega \times(0, T)$ is a line segment contained inside $\Omega \times(0, T)$. In the case $n=2$ this result is generalized to the case of analytic solutions of parabolic equations with analytic coefficients defined in an arbitrary bounded simply connected cylinder $D \times(0, T)$ where the solution vanishes on a portion of $\partial D \times(0, T)$.
\end{abstract}

I. Introduction. A classical result in potential theory is the Schwarz reflection principle for solutions of Laplace's equation which vanish on a portion of a plane or sphere. In particular if $u$ is a solution of

$$
\Delta_{n} u=0
$$

defined for $x_{1} \geqslant 0, \mathbf{x}=\left(x_{1}, \ldots, x_{n}\right) \in \mathbf{R}^{n}$, and satisfying the boundary condition

$$
u\left(0, x_{2}, \ldots, x_{n}\right)=0 \text {, }
$$

then $u$ can be continued across $x_{1}=0$ by the formula

$$
u\left(-x_{1}, x_{2}, \ldots, x_{n}\right)=-u\left(x_{1}, x_{2}, \ldots, x_{n}\right),
$$

whereas if $u$ is a solution of (1.1) defined for $|\mathbf{x}|<a$ and vanishing on a portion $\sigma$ of the sphere $r=|\mathbf{x}|=a$ then $u$ can be continued across $\sigma$ by the formula

$$
u(r, \theta)=-\left(\frac{a}{r}\right)^{n-2} u\left(\frac{a^{2}}{r}, \theta\right)
$$

where $(r, \theta)=\left(r, \theta_{1}, \ldots, \theta_{n-1}\right)$ are spherical coordinates. Both of these results can easily be verified by observing that (1.3) and (1.4) both serve to define a harmonic function in the original domain of definition of $u$ together with its "reflection" in such a way that $u$ is continuously differentiable across the common boundary of these domains. Hence $u$ is in fact harmonic in the union of these two domains and their common boundary and by Holmgren's uniqueness theorem [3] this continuation is unique.

It is the purpose of this note to extend the above reflection principles to the case of parabolic equations, in particular for the heat equation

$$
\Delta_{n} u=u_{t}
$$

defined in a cylindrical domain of the form $\Omega \times(0, T)$ where $T>0$ and $\partial \Omega$ either

Received by the editors July 6, 1979 and, in revised form, February 20, 1980.

1980 Mathematics Subject Classification. Primary 35B60; Secondary 35K 10.

${ }^{1}$ This research was supported in part by NSF Grant MCS78-02452 and AFOSR Grant 76-2879. 
contains a portion of the plane $x_{1}=0$ on which $u$ vanishes for $0<t<T$, or $\Omega$ is a ball and $u$ vanishes on $\partial \Omega \times(0, T)$. The first problem is trivial since in this case $u$ can be continued by the reflection formula

$$
u\left(-x_{1}, x_{2}, \ldots, x_{n}, t\right)=-u\left(x_{1}, x_{2}, \ldots, x_{n}, t\right),
$$

the proof being identical to that for Laplace's equation. The case when $\Omega$ is a ball is more difficult since a reflection formula analogous to (1.4) no longer defines a solution of the heat equation for $r>a$, and hence a new reflection formula must be derived. This we shall do in §III of this note, basing our result on a new class of integral representations for solutions of the heat equation developed in §II. In §IV we shall indicate how our results can be considerably generalized in the case when $n=2$. A noteworthy result of this analysis is that the domain of dependence of the value of a solution of the heat equation at a point outside the cylinder $\Omega \times(0, T)$ is a line segment contained inside $\Omega \times(0, T)$. This is in contrast to the reflection rule (1.4) for solutions of Laplace's equation, where the domain of dependence is simply the image point under the Kelvin inversion (1.4).

We note that if $\Omega$ is the ball $\{\mathbf{x}:|x|<a\}$ and $u$ is a solution of (1.5) in $\Omega \times(0, T)$, continuous in $\bar{\Omega} \times(0, T)$, and vanishing on $\partial \Omega \times(0, T)$, then $u$ is an analytic function of $x$ and $t$ in $\bar{\Omega} \times(0, T)$ [2, p. 212]. Hence without loss of generality we can restrict our attention to analytic solutions of the heat equation.

II. Integral representations of solutions to the heat equation in a cylinder. Let $\Omega=\{\mathbf{x}:|\mathbf{x}| \leqslant a\}$ and $u$ a solution of $(1.5)$ in $\Omega \times(0, T)$ such that $u$ is continuous in $\bar{\Omega} \times(0, T)$ and vanishes on $\partial \Omega \times(0, T)$. We shall show that there exists a kernel $K$ and harmonic function $h$ depending analytically on a parameter $t$, defined in $\bar{\Omega} \backslash\{0\} \times(0, T)$, and vanishing on $\partial \Omega \times(0, T)$, such that in the punctured cylinder $\Omega \backslash\{0\} \times(0, T) u$ can be represented in the form

$$
\begin{aligned}
u(r, \theta, t) & =(\mathrm{I}+\mathrm{T}) h \\
& =h(r, \theta, t)+\frac{1}{2 \pi i} \oint_{|t-\tau|=\delta} \int_{a}^{r} s^{n-3} K(r, s, t-\tau) h(s, \theta, \tau) d s d \tau,
\end{aligned}
$$

where $\delta>0$ is arbitrarily small. Indeed, substituting (2.1) into (1.5) and integrating by parts shows (see the Appendix) that $u$ will be a solution of (1.5) in $\Omega \backslash\{0\} \times$ $(0, T)$, vanishing on $\partial \Omega \times(0, T)$, provided $K(r, s, t)$ satisfies the differential equation

$$
r^{2}\left[K_{r r}+\frac{n-1}{r} K_{r}\right]-s^{2}\left[K_{s s}+\frac{n-1}{s} K_{s}\right]=r^{2} K_{t}
$$

and the initial conditions

$$
\begin{aligned}
& K(r, a, t)=0, \\
& K(r, r, t)=r^{2-n}\left(r^{2}-a^{2}\right) / 4 t^{2} .
\end{aligned}
$$

To establish the existence of the operator $\mathbf{I}+\mathbf{T}$ we now show that an (analytic) solution of (2.2), (2.3) exists for $0<r<s<a, a<s<r<\infty$, and $|t|>0$. To this end we make the change of variables

$$
\zeta=\sqrt{r s}, \quad \eta=\sqrt{r / s}
$$


and reduce the initial value problem (2.2), (2.3) to

$$
\begin{aligned}
M_{\zeta \eta}-\zeta \eta M_{t} & =0, \\
M(a \eta, \eta, t) & =0, \\
M(\zeta, 1, t) & =\left(\zeta^{2}-a^{2}\right) / 4 t^{2}
\end{aligned}
$$

where

$$
M(\zeta, \eta, t)=\zeta^{n-2} K(\zeta \eta, \zeta / \eta, t),
$$

and look for a solution of (2.5), (2.6) for $|t|>0$ and $(\zeta, \eta)$ in the shaded region of Figure 1 below.

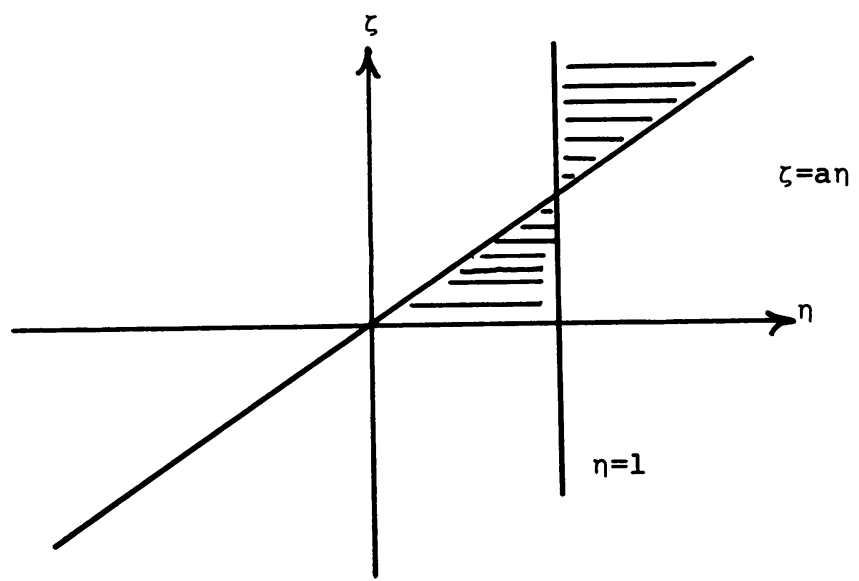

Figure 1

We seek a solution of (2.5), (2.6) in the form

$$
M(\zeta, \eta, t)=\sum_{k=1}^{\infty} \frac{a^{(k)}(\zeta, \eta)}{t^{k+1}}
$$

where the $a^{(k)}$ are to be determined, and find that for (2.8) to be a solution of (2.5), (2.6) the $a^{(k)}$ must satisfy the recursion scheme

$$
\begin{aligned}
a^{(1)} & =\frac{1}{4}\left(\zeta^{2}-a^{2} \eta^{2}\right) ; \\
a_{\xi \eta}^{(k)} & =-k \zeta \eta a^{(k-1)}, \quad k>2 ; \\
a^{(k)}(\zeta, 1) & =a^{(k)}(a \eta, \eta)=0, \quad k>2 .
\end{aligned}
$$

Hence

$$
a^{(k)}(\zeta, \eta)=-k \int_{1}^{\eta} \int_{a \eta}^{\zeta} a^{(k-1)}(\sigma, \rho) \sigma \rho d \sigma d \rho, \quad k>2,
$$

and due to the double integral appearing in (2.10) we can conclude that $a^{(k)}=$ $O(1 / k !)$, thus implying that $(2.8)$ is convergent for $|t|>0$ and $(\zeta, \eta)$ in the shaded region of Figure 1 . It is an easy matter to verify the analyticity of $M$ and the fact that (2.8) indeed defines a solution of (2.5), (2.6). Thus we have established the existence of the operator $\mathbf{I}+\mathbf{T}$.

The above analysis shows that $(I+T) h$ defines an analytic solution of the heat equation in $\Omega \backslash\{0\} \times(0, T)$ vanishing on $\partial \Omega \times(0, T)$. It remains to be shown that 
any such $u$ can be represented in this form, and to this end we construct an integral representation for the inverse operator $(I+T)^{-1}$. In particular we look for a kernel $E$ such that

$$
\begin{aligned}
h(r, \theta, t) & =(\mathbf{I}+\mathbf{T})^{-1} u \\
& =u(r, \theta, t)+\frac{1}{2 \pi i} \oint_{|t-\tau|=\delta} \int_{a}^{r} s^{n-3} E(r, s, t-\tau) u(s, \theta, \tau) d s d \tau
\end{aligned}
$$

where $h$ and $u$ are defined as before. Substituting (2.11) into Laplace's equation (1.1) and integrating by parts shows that $h$ will be a solution of (1.1) in $\Omega \backslash\{0\} \times$ $(0, T)$ (depending analytically on the parameter $t$ ) and vanishing on $\partial \Omega \times(0, T)$ provided $E(r, s, t)$ satisfies the differential equation

$$
r^{2}\left[E_{r r}+\frac{n-1}{r} E_{r}\right]-s^{2}\left[E_{s s}+\frac{n-1}{s} E_{s}\right]=-s^{2} E_{t}
$$

and the initial conditions

$$
\begin{aligned}
& E(r, a, t)=0, \\
& E(r, r, t)=-r^{2-n}\left(r^{2}-a^{2}\right) / 4 t^{2} .
\end{aligned}
$$

Making the change of variables (2.4) shows that

$$
M(\zeta, \eta, t)=\zeta^{n-2} E(\zeta \eta, \zeta / \eta, t)
$$

satisfies the initial value problem

$$
\begin{aligned}
M_{\zeta \eta}+\frac{\zeta}{\eta^{3}} M_{t} & =0, \\
M(a \eta, \eta, t) & =0, \\
M(\zeta, 1, t) & =-\left(\zeta^{2}-a^{2}\right) / 4 t^{2},
\end{aligned}
$$

and the existence of a solution to this problem follows in the same manner as that of (2.5), (2.6). The fact that the operator defined by the right hand side of (2.11) is indeed $(I+T)^{-1}$ follows from the fact that the initial conditions (2.3a) and (2.13a) imply that $h_{r}(a, \theta, t)=u_{r}(a, \theta, t)$, i.e. $h$ and $u$ have identical Cauchy data on $r=a$. Hence if $h$ is defined by (2.11) for a given $u$ satisfying the heat equation in $\Omega \times(0, T)$ and vanishing on $\partial \Omega \times(0, T)$, then by Holmgren's uniqueness theorem $u$ has the representation (2.1). We have now established the existence of the operator $(\mathbf{I}+\mathbf{T})^{-1}$.

III. The Schwarz reflection principle for the heat equation. We are now in a position to prove the following theorem.

TheOREM. Let $\Omega=\{\mathbf{x}:|\mathbf{x}|<a\}, T>0$, and $u$ a solution of the heat equation (1.5) in $\Omega \times(0, T)$ such that $u$ is continuous in $\bar{\Omega} \times(0, T)$ and vanishes on $\partial \Omega \times(0, T)$. Then $u$ can be uniquely continued as a solution of the heat equation into all of $\mathbf{R}^{n} \times(0, T)$ where the domain of dependence of $u$ at the point $(r, \theta, t)$ for $r>a$ is the line segment joining $\left(a^{2} / r, \theta, t\right)$ to $(a, \theta, t)$.

Proof. Define the harmonic function $h$ depending analytically on the parameter $t$ by

$$
h=(\mathbf{I}+\mathbf{T})^{-1} u
$$


Then, for each fixed $t, h$ is harmonic in $\Omega \backslash\{0\}$, continuous in $\bar{\Omega} \backslash\{0\}$ and satisfies

$$
h(a, \theta, t)=0 \text {. }
$$

Hence from (1.4) $h$ can be continued across $r=a$ by the formula

$$
h(r, \theta, t)=-\left(\frac{a}{r}\right)^{n-2} h\left(\frac{a^{2}}{r}, \theta, t\right),
$$

and this continuation clearly preserves the analytic dependence of $h$ on the parameter $t$. The fact that $u$ can be continued into all of $\mathbf{R}^{n} \times(0, T)$ now follows from (3.3), the regularity of the kernel $K$, and the representation (2.1). Indeed, (2.1), (2.11) and (3.3) can be combined together to provide a specific "reflection formula" for this continuation. In particular it follows from (2.1), (2.11) and (3.5) that the value of $u$ at a point $(r, \theta, t)$ outside the cylinder $\Omega \times(0, t)$ depends continuously on the value of $u$ and its derivatives with respect to time along the line segment joining $\left(a^{2} / r, \theta, t\right)$ to $(a, \theta, t)$ (see Figure 2$)$.

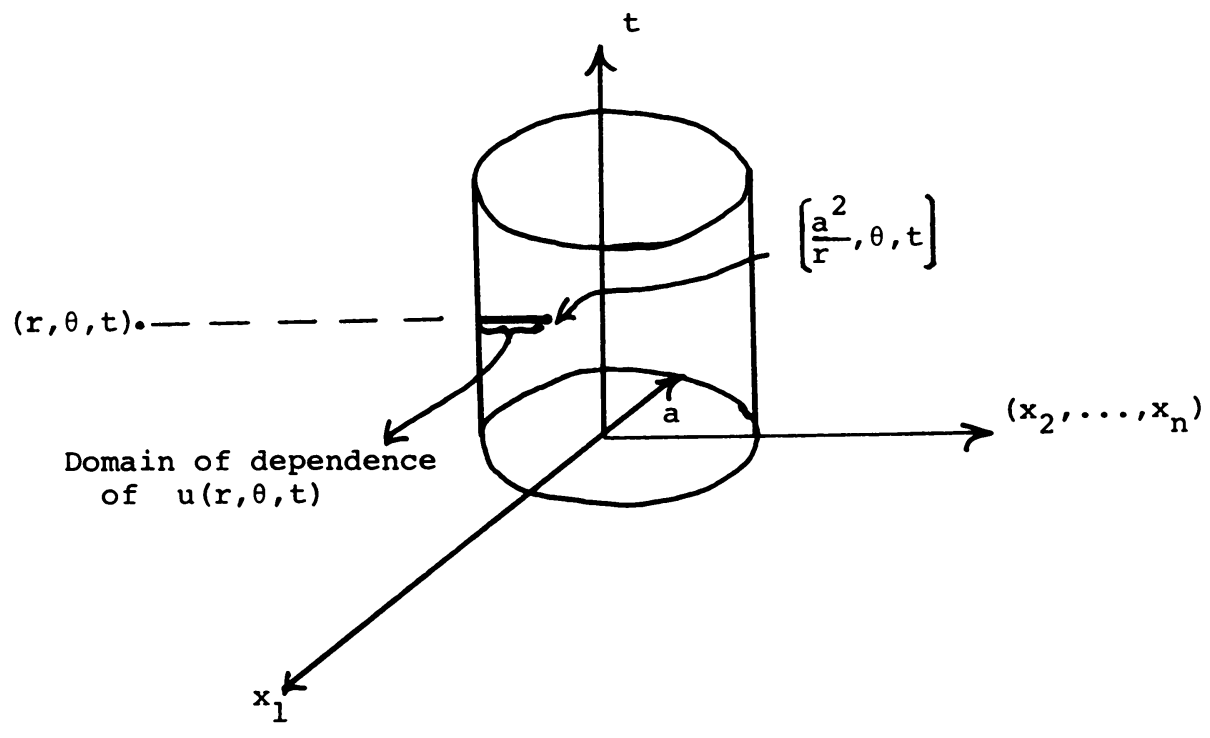

Figure 2

IV. Remarks on the case of two space variables. In the case of two space variables the above results can be considerably generalized. In particular consider the parabolic equation

$$
\Delta_{2} u+a(x, y, t) u_{x}+b(x, y, t) u_{y}+c(x, y, t) u=d(x, y, t) u_{t}
$$

defined in a cylinderical domain $D \times(0, T)$ where $D$ is a bounded simply connected domain such that $u$ vanishes on a portion $\sigma$ of $\partial D \times(0, T)$ and the coefficients of (4.1) are assumed to be analytic functions of their independent variables in a sufficiently large domain. By the use of a conformal mapping we can assume without loss of generality that $\sigma$ is a portion of the $(y, t)$ plane and we make the assumption that $u$ is an analytic function of $x, y$ and $t$ in $\bar{D} \times(0, T)$. In order to continue $u$ across $\sigma$ into the mirror image of $D \times(0, T)$ we modify 
Garabedian's proof of the reflection principle for elliptic equations in two independent variables $[3, \S 16.4]$. The first step in this approach is to keep $t=t_{0} \in(0, T)$ fixed and $x=x_{1}$ real, but allow $y=y_{1}+i y_{2}$ to be complex and to continue $u$ off the real domain into the wedge $-x_{1}<y_{2}<x_{1}, x_{1}>0$, where $y_{1}$ appears merely as a parameter. This continuation can be found by solving the Cauchy problem

$$
\begin{gathered}
u_{x_{1} x_{1}}-u_{y_{2} y_{2}}+a u_{x_{1}}-i b u_{y_{2}}+c u=d u_{t}, \\
u\left(x_{1}, y_{1}, 0, t\right)=u\left(x_{1}, y_{1}, t\right), \\
u_{y_{2}}\left(x_{1}, y_{1}, 0, t\right)=i u_{y_{1}}\left(x_{1}, y_{1}, t\right) .
\end{gathered}
$$

The solution to initial value problems of this type was discussed in [1], and, under the assumption that $u$ is an analytic function of $t$, it follows from this analysis that a solution to (4.2a), (4.2b) can be found by successive approximations for $-x_{1}<y_{2}$ $\left\langle x_{1},\left|t-t_{0}\right|<\delta\right.$, where $\delta>0$. The next step is to solve a Goursat problem for (4.2a) on each of the angular regions $y_{2}>x_{1}>0$ and $y_{2}<-x_{1}<0$ (see Figure 3).

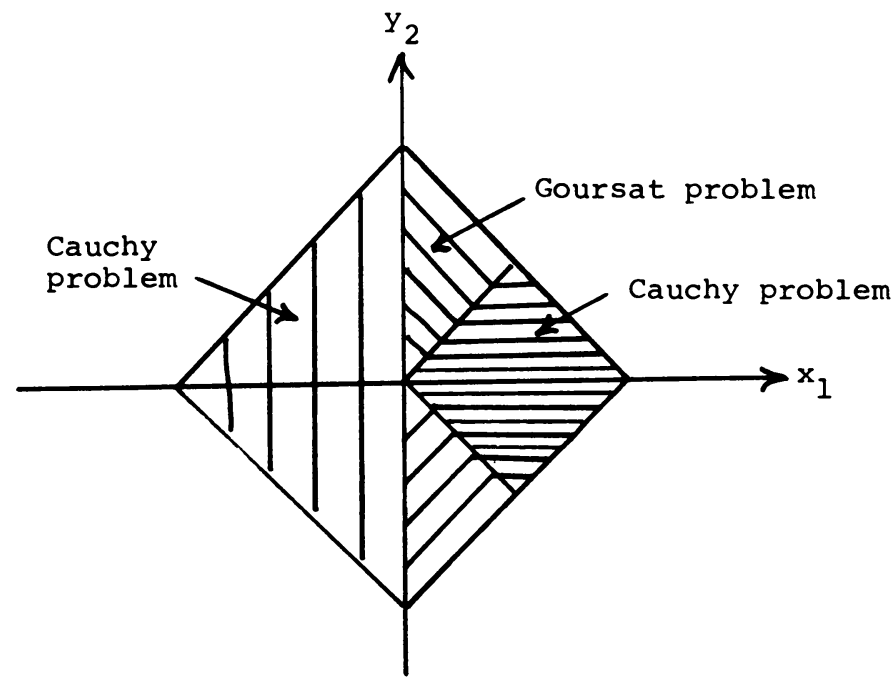

Figure 3

The solution to these problems can again be obtained by successive approximations [1]. We can now determine $u_{x_{1}}\left(0, y_{1}, y_{2}, t\right)$ and, following the analysis of Garabedian, show that this function is analytic for $\left|t-t_{0}\right|<\delta$ and $y_{1}+i y_{2}$ in $D$ (where $D$ is now considered as a domain in the complex plane). The final step is to continue $u$ across $\sigma$ by using the boundary condition $u\left(0, y_{1}, y_{2}, t\right)=0$ and solving a Cauchy problem for (4.2a) with data prescribed on the plane $x_{1}=0$. This now provides a continuation of $u$ into the mirror image of $D \times(0, T)$ with respect to $\sigma$, except for those points which cannot be reconnected to $\sigma$ by perpendicular line segments. Note that the domain of dependence of a point in the mirror image is a line segment in $D \times(0, T)$ joining the image point to $\sigma$. For more details of the above continuation procedure we refer the reader to the proof for the time independent case in [3]. 
We note that under the assumption that $u$ can indeed be continued across $\sigma$ (the proof of which is outlined above), explicit reflection formulas can be obtained by using the approach developed by Hill in [4].

Appendix. We show here that under the assumptions (2.2), (2.3) we have that (2.1) defines a solution of (1.5). In spherical coordinates (1.5) has the form

$$
L[u] \equiv \frac{\partial^{2} u}{\partial r^{2}}+\frac{n-1}{r} \frac{\partial u}{\partial r}+\frac{1}{r^{2}} \Lambda u-\frac{\partial u}{\partial t}=0
$$

where $\Lambda$ is an operator depending only on the angular coordinates (cf. [5, p. 293]). Hence from (2.1), (2.3b) we have

$$
\begin{aligned}
L[u]= & -\frac{\partial h}{\partial t}+\frac{1}{2 \pi i} \oint_{|t-\tau|=\delta} \int_{a}^{r} s^{n-3}\left[\frac{\partial^{2} K}{\partial r^{2}}+\frac{n-1}{r} \frac{\partial K}{\partial r}-\frac{\partial K}{\partial t}\right] h(s, \theta, \tau) d s d \tau \\
& +\frac{1}{2 \pi i} \oint_{|t-\tau|=\delta}\left[\frac{(n-1)\left(1-a^{2} / r^{2}\right)+\left(1+a^{2} / r^{2}\right)}{4(t-\tau)^{2}}\right] h(r, \theta, \tau) d \tau \\
& +\frac{1}{2 \pi i} \oint_{|t-\tau|=\delta}\left[\frac{r-a^{2} / r}{4(t-\tau)^{2}}\right] \frac{\partial h}{\partial r}(r, \theta, \tau) d \tau \\
& +\frac{1}{2 \pi i} \oint_{|t-\tau|=\delta} r^{n-3} K(r, r, t-\tau) h(r, \theta, \tau) d \tau \\
& +\frac{1}{2 \pi i r^{2}} \oint_{|t-\tau|=\delta} \int_{a}^{r} s^{n-3} K(r, s, t-\tau) \Lambda h(s, \theta, \tau) d s d \tau
\end{aligned}
$$

But integrating by parts and using (2.3a) gives

$$
\begin{aligned}
\frac{1}{2 \pi i r^{2}} \oint_{|t-\tau|=\delta} & \int_{a}^{r} s^{n-3} K(r, s, t-\tau) \Lambda h(s, \theta, \tau) d s d \tau \\
= & -\frac{1}{2 \pi i r^{2}} \oint_{|t-\tau|=\delta} \int_{a}^{r} s^{n-3} K(r, s, t-\tau)\left[s^{2} \frac{\partial^{2} h}{\partial s^{2}}+(n-1) s \frac{\partial h}{\partial s}\right] d s d \tau \\
= & -\frac{1}{2 \pi i} \oint_{|t-\tau|=\delta}\left[\frac{r-a^{2} / r^{2}}{4(t-\tau)^{2}}\right] \frac{\partial h}{\partial r}(r, \theta, \tau) d \tau \\
& +\frac{1}{2 \pi i} \oint_{|t-\tau|=\delta} r^{n-3} \frac{\partial K}{\partial s}(r, r, t-\tau) h(r, \theta, \tau) d \tau \\
& -\frac{1}{2 \pi i r^{2}} \oint_{|t-\tau|=\delta} \int_{a}^{r} s^{n-3}\left[s^{2} \frac{\partial^{2} K}{\partial s^{2}}+(n-1) s \frac{\partial K}{\partial s}\right] h(s, \theta, \tau) d s d \tau .
\end{aligned}
$$

From (2.3b) we have

$$
r^{n-3} \frac{\partial K}{\partial s}(r, r, t)=\frac{(4-n)-(2-n) a^{2} / r^{2}}{4 t^{2}}-r^{n-3} \frac{\partial K}{\partial r}(r, r, t) .
$$


Putting (1), (2) and (3) together gives, using (2.2),

$$
\begin{aligned}
& L[u]=-\frac{\partial h}{\partial t} \\
&+\frac{1}{2 \pi i r^{2}} \oint_{|t-\tau|=\delta} \int_{a}^{r} s^{n-3}\left[r^{2} \frac{\partial^{2} K}{\partial r^{2}}+(n-1) r \frac{\partial K}{\partial r}-s^{2} \frac{\partial^{2} K}{\partial s^{2}}\right. \\
&\left.-(n-1) s \frac{\partial K}{\partial s}-r^{2} \frac{\partial K}{\partial t}\right] h(s, \theta, \tau) d s d \tau \\
&+\frac{1}{2 \pi i} \oint_{|t-\tau|=\delta} \frac{1}{(t-\tau)^{2}} h(r, \theta, \tau) d \tau=0
\end{aligned}
$$

which is the desired result.

\section{REFERENCES}

1. D. Colton, Integral operators and reflection principles for parabolic equations in one space variable, J. Differential Equations 15 (1974), 551-559.

2. A. Friedman, Partial differential equations, Holt, Rinehart and Winston, New York, 1969.

3. P. R. Garabedian, Partial differential equations, Wiley, New York, 1964.

4. C. D. Hill, A method for the construction of reflection laws for a parabolic equation, Trans. Amer. Math. Soc. 133 (1968), 357-372.

5. I. N. Vekua, New methods for solving elliptic equations, Wiley, New York, 1967.

Department of Mathematical Sciences, University Of Delaware, Newark, Delaware 19711 